\title{
Resultados del reemplazo valvular aórtico con prótesis mecánica en el Instituto Nacional del Tórax
}

\author{
Mauricio Villavicencio T, Eduardo Turner G, \\ Lorenzo Naranjo T.
}

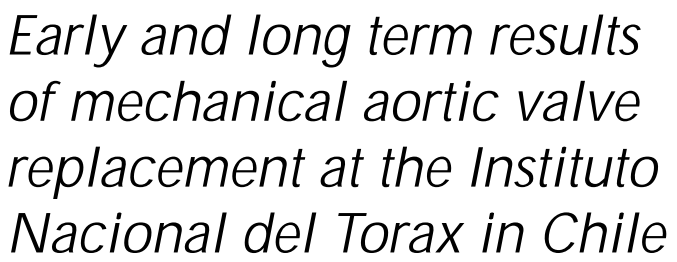

Background: Mechanical aortic valve replacement (AVR) results have been published extensively in industrialized countries. Aim: To assess our immediate and late results in patients subjected to AVR. Patients and Methods: We retrospectively studied 194 patients subjected to isolated AVR between 1995 and 2003. Mean age was $57 \pm 13$ years and 119 (61\%) were male. One hundred thirty nine (73\%) were in functional class III-IV, 20 (10\%) had a previous cardiac operation and 25 (13\%) were operated as an emergency. Surgical indication was stenosis in 110 (58\%), regurgitation in $49(26 \%)$ and stenosis/regurgitation in $31(16 \%)$. Etiology was bicuspid valve 56 (29\%), degenerative lesions $55(28 \%)$, rheumatic valve disease $38(20 \%)$ and endocarditis $27(14 \%)$. Medtronic Hall was the most common prosthesis used in 157 patients (81\%). Mean cardiopulmonary bypass time $97 \pm 29$ min and mean cross clamp time was $69 \pm 21$ min. Results: Operative mortality was $4.6 \%$ (3\% in elective surgery, $16 \%$ in emergency surgery and $0 \%$ in reoperations). Follow-up was complete in $100 \%$ of cases, totalizing 636 patients-year. Survival was $91 \pm 2 \%, 80 \pm 4 \%$ and $73 \pm 6 \%$, at 1,5 and 7 years, respectively. Multivariate risk analysis identified renal failure and endocarditis as predictors of early and late mortality. During follow up, the linear incidence rate for hemorrhage was 3.29\% /patients-year, thromboembolism $2.04 \%$ patients-year and endocarditis $1.1 \%$ patients-year. Conclusions: AVR has low overall and elective mortality. Midterm survival is good but linear event rates related to anticoagulant treatment are higher than those previously published in industrialized countries. Renal failure and endocarditis were risk factors for early and late death (Rev Méd Chile 2005; 133: 1161-72).

(Key Words: Aortic valve; Endocarditis; Heart valve diseases; Rheumatic fever)

Recibido el 5 de octubre, 2004. Aceptado el 3 de junio, 2005.

Servicio de Cirugía Cardiovascular, Instituto Nacional del Tórax, Santiago, Chile.

Correspondencia a: Dr. Mauricio Villavicencio. José Miguel Infante 717, Providencia, Santiago. Chile. Fax: 56-23403539. E-mail: mauriciovillavicencio@yahoo.com 
$\mathrm{E}^{1}$ reemplazo valvular aórtico es una operación frecuente. En pacientes jóvenes, la opción más popular es una prótesis mecánica que requiere tratamiento anticoagulante permanente. De acuerdo a la literatura médica internacional, los resultados de esta operación son satisfactorios y generalmente predecibles, con factores de riesgo para complicaciones claramente identificados. Sin embargo, la mayoría de las publicaciones dedicadas a este tema se origina en países con realidades socioeconómicas mucho mejores que las que tiene Chile y, por lo tanto, no son extrapolables a nuestra realidad ${ }^{1-4}$.

El Instituto Nacional del Tórax de Santiago, Chile, es un hospital público que atiende pacientes de un nivel socioeconómico bajo. Muchos de estos pacientes viven lejos de centros de atención médica. El primer objetivo de esta publicación es evaluar los resultados del reemplazo valvular aóntico aislado con prótesis mecánica en cuanto a morbimortalidad perioperatoria, sobrevida alejada y libre de eventos de los pacientes operados en el Instituto

Tabla 1. C aracterísticas preoperatorias

\begin{tabular}{|c|c|c|c|}
\hline \multicolumn{2}{|l|}{ Variable } & Júmero & Porcentaje \\
\hline \multicolumn{2}{|l|}{ Número total } & 194 & \\
\hline \multicolumn{2}{|l|}{ Edad promedio (años) } & \multicolumn{2}{|c|}{$56,8 \pm 12,9$} \\
\hline \multicolumn{2}{|l|}{ Mayor de 65 años } & 60 & $31 \%$ \\
\hline \multicolumn{2}{|l|}{ Hombres } & 119 & $61 \%$ \\
\hline \multicolumn{2}{|l|}{ Mujeres } & 75 & $39 \%$ \\
\hline \multicolumn{2}{|c|}{ Vive fuera de la región metropolitana } & 58 & $30 \%$ \\
\hline \multicolumn{2}{|c|}{ IMC promedio (índice de masa corporal) } & & \\
\hline \multicolumn{2}{|l|}{ Tabaquismo } & 64 & $33 \%$ \\
\hline \multicolumn{2}{|l|}{ Diabetes mellitus } & 21 & $11 \%$ \\
\hline \multicolumn{2}{|c|}{ Insuficiencia renal (creatinina $>2 \mathrm{mg} / \mathrm{dl}$ ) } & 7 & $4 \%$ \\
\hline \multirow{2}{*}{\multicolumn{2}{|c|}{$\begin{array}{l}\text { Creatinina promedio } \\
\text { Hipercolesterolemia (>200 mq/dl) }\end{array}$}} & & \\
\hline & & 26 & $18 \%$ \\
\hline \multicolumn{2}{|l|}{ Hipertensión arterial } & 76 & $39 \%$ \\
\hline \multicolumn{2}{|l|}{ Accidente vascular encefálico } & 10 & $5 \%$ \\
\hline \multicolumn{2}{|l|}{ Endocarditis infecciosa } & 27 & $14 \%$ \\
\hline \multicolumn{2}{|l|}{ Enf bronquial obstructiva crónica } & 6 & $3 \%$ \\
\hline \multicolumn{2}{|l|}{ Cirugía cardíaca previa } & 20 & $10 \%$ \\
\hline \multicolumn{2}{|l|}{ Fibrilación auricular } & 24 & $12 \%$ \\
\hline \multirow[t]{4}{*}{ Capacidad funcional (NYHA) } & I & 13 & $7 \%$ \\
\hline & II & 39 & $20 \%$ \\
\hline & III & 103 & $54 \%$ \\
\hline & IV & 36 & $19 \%$ \\
\hline \multicolumn{2}{|l|}{ Shock cardiogénico } & 6 & $3 \%$ \\
\hline \multirow{2}{*}{\multicolumn{2}{|c|}{$\begin{array}{l}\text { Uso inótropos } \\
\text { Fracción de eyección promedio }\end{array}$}} & 8 & $4 \%$ \\
\hline & & & \\
\hline \multicolumn{2}{|c|}{ Fracción de eyección menor o igual a 35\% } & 21 & $11 \%$ \\
\hline Dimensión ventricular promedio & Sistólica & & \\
\hline \multirow{3}{*}{ Indicación quirúrgica } & $\begin{array}{l}\text { Diastolica } \\
\text { Estenosis aórtica }\end{array}$ & 110 & $58 \%$ \\
\hline & Insuficiencia aórtica & 49 & $26 \%$ \\
\hline & Estenosis/insuficiencia aórtica & 31 & $16 \%$ \\
\hline \multirow[t]{2}{*}{ Carácter de la operación } & Electiva & 168 & $87 \%$ \\
\hline & Urgente & 25 & $13 \%$ \\
\hline
\end{tabular}

Los porcentajes no siempre se relacionan exactamente con el número total, dado que faltan datos en algunas variables. 
Nacional del Tórax. El segundo objetivo es analizar los factores predictores de complicaciones operatorias y alejadas mediante un análisis uni y multivariado aplicado a este mismo grupo de pacientes.

\section{PACIENTES Y MÉTODOS}

Entre noviembre de 1995 y marzo de 2003 se operaron 194 pacientes de RVA en el Instituto Nacional del Tórax, Santiago, Chile. Excluidos de esta muestra están todos aquellos con cirugía cardiovascular asociada. Todos los pacientes recibieron tratamiento anticoagulante con acenocumarol después de la operación en los policlínicos del Servicio Nacional de Salud. El análisis de la morbimortalidad perioperatoria fue retrospectivo y realizado a través de fichas clínicas y protocolos operatorios. El seguimiento se efectuó entre agosto de 2002 y septiembre de 2003, mediante contactos telefónicos, revisión de fichas clínicas y constatación de los fallecimientos en el Registro Civil. La codificación de las variables se hizo usando como pauta la base de datos de la STS (The Society of Thoracic Surgeons) y el registro de los eventos en el seguimiento se realizó de acuerdo a las normas del comité de la AATS (The American Association for Thoracic Surgery) ${ }^{5}$.

De los 194 pacientes, 119 (61\%) eran hombres y la edad promedio fue de $56,8 \pm 12,9$ años.
Cincuenta y ocho (30\%) de los pacientes vivían fuera de la Región Metropolitana. Por otro lado, 76 (39\%) pacientes sufrían de hipertensión arterial, 21 (11\%) eran diabéticos y 7 (4\%) tenían insuficiencia renal. Se operaron de urgencia 25 (13\%) y tenían endocarditis 27 (14\%). Ciento treinta y nueve (73\%) tenían capacidad funcional (NYHA) III ó IV, 21 (11\%) mostraban una fracción de eyección (FE) igual o menor a 35\% y 110 (58\%) tenían como indicación predominante la estenosis. Veinte pacientes tenían cirugía cardíaca con esternotomía previa (10\%), de los cuales $5(3 \%)$ tenían un reemplazo valvular aórtico previo y 15 (8\%) otro tipo de cirugía valvular no congénita. No hubo pacientes con cirugía coronaria previa (Tabla 1).

Las etiologías predominantes fueron bicúspide 56 (29\%), degenerativa 55 (28\%), reumática 38 (20\%), endocarditis 27 (14\%), anuloectasia 7 (4\%), mixomatosa 6 (3\%) y disfunción protésica $5(3 \%)$.

Las prótesis usadas fueron: Medtronic Hall $(\mathrm{n}=157,81 \%)$, St. Vincent $(\mathrm{n}=17,9 \%)$, Starr Edwards $(n=13,7 \%)$, Starr Machi $(n=4,2 \%)$ y ON-X $(\mathrm{n}=2,1 \%)$. Esto totaliza 174 prótesis de monodisco, 17 prótesis de bola y 2 prótesis de doble disco. En un caso no estaba espeficado en la ficha clínica el tipo de prótesis mecánica. El diámetro protésico promedio fue de $22,4 \pm 1,9$.

El tiempo promedio de pinzamiento aórtico: $69 \pm 21$ min y el de circulación extracorpórea $97 \pm$ 29 min. Tabla 2.

Tabla 2. C aracterísticas operatorias

\begin{tabular}{|llrr|}
\hline Variable & & Número & Porcentaje \\
\hline Etiología & Bicúspide & 56 & $29 \%$ \\
& Degenerativa & 55 & $28 \%$ \\
& Reumática & 38 & $20 \%$ \\
& Endocarditis & 27 & $14 \%$ \\
& Anuloectasia & 7 & $4 \%$ \\
& Mixomatosa & 6 & $3 \%$ \\
& Disfunción protésica & 5 & $3 \%$ \\
Prótesis mecánica & Medtronic Hall (monodisco) & 157 & $81 \%$ \\
& St Vincent (monodisco) & 17 & $9 \%$ \\
& Starr Edwards (bola) & 13 & $7 \%$ \\
& Starr Machi (bola) & 4 & $2 \%$ \\
Diámetro protésico promedio & ON-X (doble disco) & 2 & $1 \%$ \\
Tiempo pinzamiento aórtico promedio & & $22,4 \pm 1,9$ & \\
Tiempo circulación extracorpórea promedio & & $68,7 \pm 21,0$ & \\
\hline
\end{tabular}


Estadística: Las variables continuas fueron comparadas con el test de Student y las categóricas con el $\mathrm{x}^{2} \mathrm{o}$ bien con el de Fisher cuando los valores esperados eran pequeños. La sobrevida fue evaluada con el método de Kaplan-Meier y las curvas comparadas con el test de Log-Rank. La sobrevida se expresa en porcentaje \pm el error estándar de la media. Para el análisis de múltiples variables se empleó el modelo de regresión logística para buscar los predictores de mortalidad operatoria y el de Cox para encontrar las variables asociadas a muerte y eventos en el seguimiento. Todas las variables listadas en el apéndice fueron analizadas en forma uni y multivariada. Solamente las variables que fueron significativas están listadas en los resultados. El valor de p menor de 0,05 se consideró significativo en todos los casos. No todos los pacientes tenían el $100 \%$ de los datos disponibles en su ficha clínica por lo que el total o denominador no fue siempre 194 para el cálculo de los porcentajes respectivos en cada una de las variables.

\section{RESULTADOS}

La mortalidad operatoria fue 4,6\%. Sin embargo, en cirugía electiva fue $3 \%$, en urgencias $16 \%$, en re-esternotomía $0 \%$ (cirugía cardíaca previa), en la segunda mitad del periodo de estudio $1 \%$ y con FE igual o menor a 35\%, 4,5\%. Las causas de muerte fueron cardiovasculares en 4 e infecciosas en 5 casos. Tabla 3.

El análisis univariado demostró que la insuficiencia renal, endocarditis infecciosa, uso de inótropos, cirugía de urgencia, presencia de insuficiencia aórtica severa y haber sido operado en la primera mitad del periodo de estudio fueron predictores de mortalidad perioperatoria. En cambio, en el análisis multivariado sólo la insuficiencia renal y la endocarditis infecciosa estuvieron asociadas a mayor mortalidad. Tabla 4.

Se tuvieron que re-explorar por hemorragia 11 (6\%) y por disfunción protésica 2 (1\%). No hubo reexploraciones por mediastinitis, sin embargo, 5 pacientes (3\%) desamollaron infección superficial de la herida operatoria. Presentaron infarto penioperatorio 1 $(0,5 \%)$, insuficiencia renal aguda $13(7 \%)$ y accidente vascular encefálico 8 (4\%). Tuvieron fibrilación auricular postoperatoria 36 pacientes (19\%). En relación a las complicaciones infecciosas no relacionadas con la herida operatoria, $9(5 \%)$ casos de neumonía y 5 (3\%) de sepsis. Por otra parte, 21 (11\%) estuvieron en ventilación mecánica más de $24 \mathrm{~h}$. El tiempo promedio de estadía en la Unidad de Cuidados Intensivos alcanzó a 3,0 \pm 3,7 días y el de estadía hospitalaria fue de 11,4 \pm 7,7 días (Tabla 5).

El seguimiento se completó en $100 \%$ para la sobrevida, siendo el promedio de $39,3 \pm 26,5$ meses. El total de seguimiento fue de 636 años-paciente. La sobrevida, incluyendo la mortalidad operatoria, fue de $91 \pm 2 \%$ (1 año), $80 \pm 4 \%$ (5 años) y $73 \pm 6 \%$ ( 7

Tabla 3. M ortalidad operatoria

\begin{tabular}{|llrrr|}
\hline Variable & Número & Total & Porcentaje \\
\hline Mortalidad operatoria & Global & 9 & 194 & $4,6 \%$ \\
& Cirugía electiva & 5 & 168 & $3,0 \%$ \\
& Cirugía urgencia & 4 & 25 & $16,0 \%$ \\
& Reesternotomía (cirugía cardíaca previa) & 0 & 20 & $0,0 \%$ \\
& Fracción de eyección menor a 35\% & 1 & 21 & $4,8 \%$ \\
& Operación después del & 1 & 96 & $1,0 \%$ \\
& 14 de septiembre de 1999 & & & \\
Causas de muerte & (segunda mitad del periodo de estudio) & & \\
& sepsis (origen desconocido) & 3 & & \\
& accidente vascular encefálico & 2 & & \\
& necrosis colon-peritonitis & 2 & & \\
& ruptura aórtica & 1 & & \\
& fibrilación ventricular & 1 & & \\
\hline
\end{tabular}


Tabla 4. Factores predictores de mortalidad operatoria

\begin{tabular}{|c|c|c|c|c|}
\hline \multirow{2}{*}{\multicolumn{2}{|c|}{ Análisis univariado: $\chi^{2}$, Fisher o $\mathrm{t}$ test }} & \multicolumn{2}{|c|}{ Mortalidad } & \multirow[t]{2}{*}{ Valor de $\mathrm{p}$} \\
\hline & & No & Sí & \\
\hline \multirow[t]{2}{*}{ Insuficiencia renal } & No & 181 & $5(3 \%)$ & $<0,001$ \\
\hline & Sí & 3 & $4(57 \%)$ & \\
\hline \multirow[t]{2}{*}{ Endocarditis infecciosa } & No & 161 & $4(2 \%)$ & $<0,001$ \\
\hline & Sí & 22 & $5(19 \%)$ & \\
\hline \multirow[t]{2}{*}{ Uso de inótropos } & No & 177 & $6(3 \%)$ & 0,003 \\
\hline & Sí & 6 & $2(25 \%)$ & \\
\hline \multirow[t]{2}{*}{ Cirugía de urgencia } & No & 163 & $5(3 \%)$ & 0,004 \\
\hline & Sí & 21 & $4(16 \%)$ & \\
\hline \multirow[t]{2}{*}{ Insuficiencia aórtica severa } & No & 135 & $3(2 \%)$ & 0,010 \\
\hline & Sí & 50 & $6(11 \%)$ & \\
\hline \multirow{2}{*}{$\begin{array}{l}\text { Operación en la primera mitad del } \\
\text { estudio (antes del 14/09/1999) }\end{array}$} & No & 96 & $1(1 \%)$ & 0,017 \\
\hline & Sí & 89 & $8(8 \%)$ & \\
\hline \multicolumn{5}{|c|}{ Análisis de múltiples variables: regresión logística } \\
\hline Variable & Valor de $\mathrm{p}$ & $\operatorname{EXP}(ß)$ & Inferior & Superior \\
\hline Insuficiencia renal & 0,009 & 25,18 & 2,21 & 286,67 \\
\hline Endocarditis infecciosa & 0,028 & 11,52 & 1,31 & 101,47 \\
\hline
\end{tabular}

Tabla 5. M orbilidad operatoria

\begin{tabular}{|lcc|}
\hline Variable & Número & Porcentaje \\
\hline Re-exploración por hemorragia & 11 & $6 \%$ \\
Re-exploración por disfunción valvular & 2 & $1 \%$ \\
Re-exploración por mediastinitis & 0 & $0 \%$ \\
Infarto perioperatorio & 1 & $0,5 \%$ \\
Insuficiencia renal (aumento creatinina $>1 \mathrm{mg} / \mathrm{dl}$ ) & 13 & $7 \%$ \\
Complicación neurológica & $11 *$ & $6 \%$ \\
Accidente vascular encefálico & 8 & $4 \%$ \\
Coma por más de 24 h & 5 & $3 \%$ \\
Accidente isquémico transitorio & 0 & $0 \%$ \\
Agitación sicomotora & 3 & $2 \%$ \\
Complicaciones de la herida operatoria & 5 & $3 \%$ \\
Infección superficial & 5 & $3 \%$ \\
Complicaciones infecciosas & 22 & $11 \%$ \\
Sepsis & 5 & $3 \%$ \\
Neumonía & 9 & $5 \%$ \\
Infección urinaria & 8 & $11 \%$ \\
Ventilación mecánica >24 horas & 21 & $1 \%$ \\
Neumotórax & 2 & $27 \%$ \\
Arritmias & 57 & $19 \%$ \\
Fibrilación/aleteo auricular & 36 & $6 \%$ \\
Taquicardia/fibrilación ventricular & 11 & $2 \%$ \\
Marcapaso permanente & 4 & \\
Tiempo promedio de estadía en intensivo (días) & $3,0 \pm 3,7$ & \\
Tiempo promedio de estadía hospitalaria (días) & $11,4 \pm 7,7$ & \\
\hline
\end{tabular}

Todos los pacientes en coma $>24 \mathrm{~h}$ tuvieron accidente vascular encefálico. 
años) (Figura 1). Se produjeron 20 muertes después del primer mes de la operación: 8 de causa cardíaca (insuficiencia, infarto o muerte súbita), 5 por complicaciones del tratamiento anticoagulante, 5 no cardíacas y 2 por endocarditis protésica.

El análisis univariado identificó a la diabetes, insuficiencia renal, endocarditis infecciosa, cirugía de urgencia e índice de masa corporal menor de 20 como predictores de mortalidad en el seguimiento. Sin embargo, en el multivariado sólo la insuficiencia renal y la diabetes estuvieron asociadas a menor sobrevida (Tabla 6).

El seguimiento clínico de los eventos se completó en $86,4 \%$, con un total de 450 años-

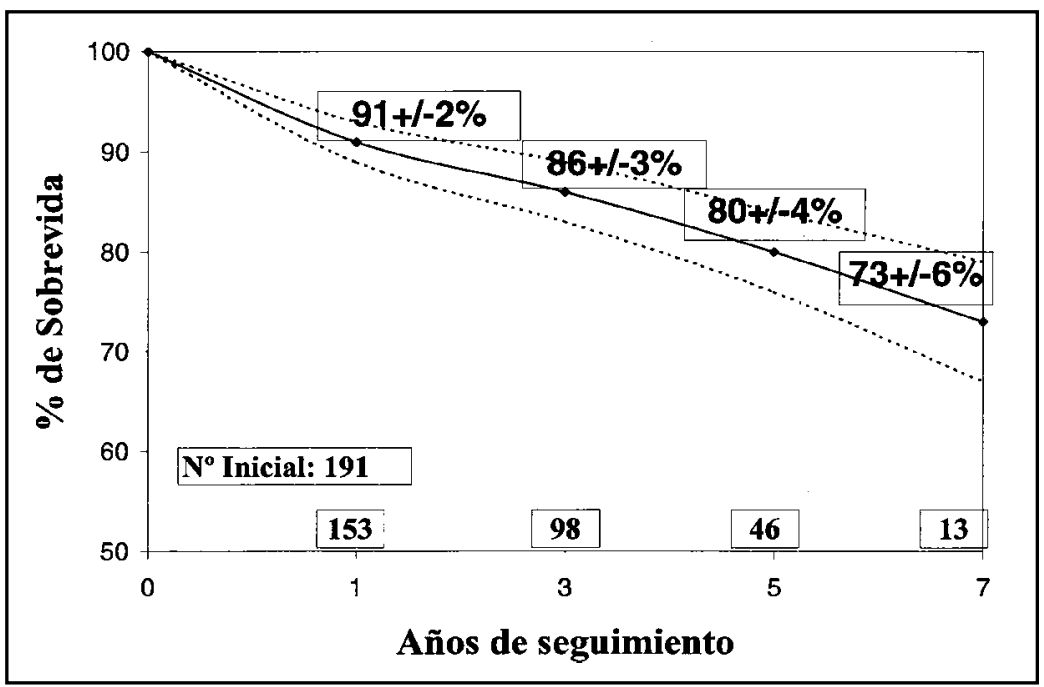

FIgURA 1. Sobrevida global

Tabla 6. Factores predictores de mortalidad en el seguimiento

\begin{tabular}{|c|c|c|c|c|}
\hline \multicolumn{5}{|c|}{ Análisis univariado: Kaplan-Meier, Log Rank } \\
\hline & Mortalidad & $\mathrm{N}^{\circ}$ eventos & $\mathrm{N}^{\circ}$ censado & Valor de $\mathrm{p}$ \\
\hline \multirow[t]{2}{*}{ Diabetes } & No & 25 & 144 & 0,019 \\
\hline & $\mathrm{Sí}$ & $\begin{array}{r}7 \\
27\end{array}$ & 14 & < 0001 \\
\hline Insuficiencia renal & Sí & 5 & 2 & $-0,001$ \\
\hline \multirow{2}{*}{$\begin{array}{l}\text { Indice de masa corporal } \\
\text { menor de } 20\end{array}$} & No & 29 & 183 & 0,043 \\
\hline & $\mathrm{Si}$ & 3 & 8 & \\
\hline \multirow[t]{2}{*}{ Endocarditis infecciosa } & No & 25 & 174 & $<0,001$ \\
\hline & Sí & 8 & 19 & \\
\hline \multirow[t]{2}{*}{ Operacion de urgencia } & $\begin{array}{l}\text { No } \\
\text { Si }\end{array}$ & 24 & 165 & 0,040 \\
\hline & $\mathrm{Si}$ & 8 & 17 & \\
\hline \multicolumn{5}{|c|}{ Análisis de múltiples variables: regresión Cox } \\
\hline \multicolumn{3}{|c|}{. } & \multicolumn{2}{|c|}{ Intervalo confianza $95 \%$ para $\operatorname{EXP}(\Omega)$} \\
\hline Insuficiencia renal & $<0,001$ & 13,51 & 4,49 & 40,64 \\
\hline Diabetes & 0,023 & 2,92 & 1,16 & 7,38 \\
\hline
\end{tabular}


paciente. La tasa lineal de hemorragia por paciente-año: 3,29\%, de tromboembolismo 2,04\% y de endocarditis protésica 1,1\%. El índice compuesto de hemorragia y tromboembolismo alcanzó 5,33\%.

La sobrevida libre de reoperación fue de $98 \pm$ $2 \%, 95 \pm 2 \%$ y $87 \pm 8 \%$, al año, 5 y 7 años, respectivamente. En cuanto a la sobrevida libre de eventos al año, 5 y 7 años para la hemorragia fue: $97 \pm 1 \%, 91 \pm 3 \%$ y $78 \pm 11 \%$; para tromboembolismo: $98 \pm 1 \%$, $94 \pm 3 \%$ y $77 \pm 11 \%$; y para endocarditis infecciosa $98 \pm 1 \%, 95 \pm 2 \%$ y $95 \pm 2 \%$ (Figuras 2 a 5 ).

El análisis univariado identificó a endocarditis infecciosa y cirugía de urgencia como predictores de eventos en el seguimiento, sin embargo, en el multivariado sólo la endocarditis estuvo asociada (Tabla 7). El vivir fuera de la Region Metropolitana no se asoció a mayor incidencia de eventos en ambos tipos de análisis.

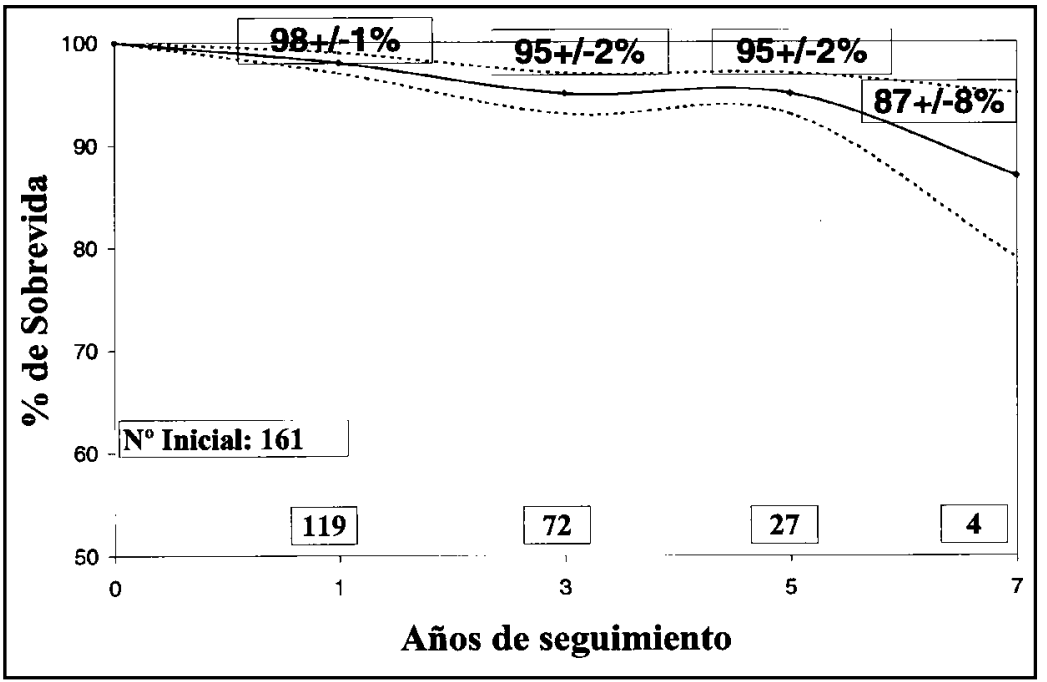

FIGURA 2. Sobrevida libre de operación

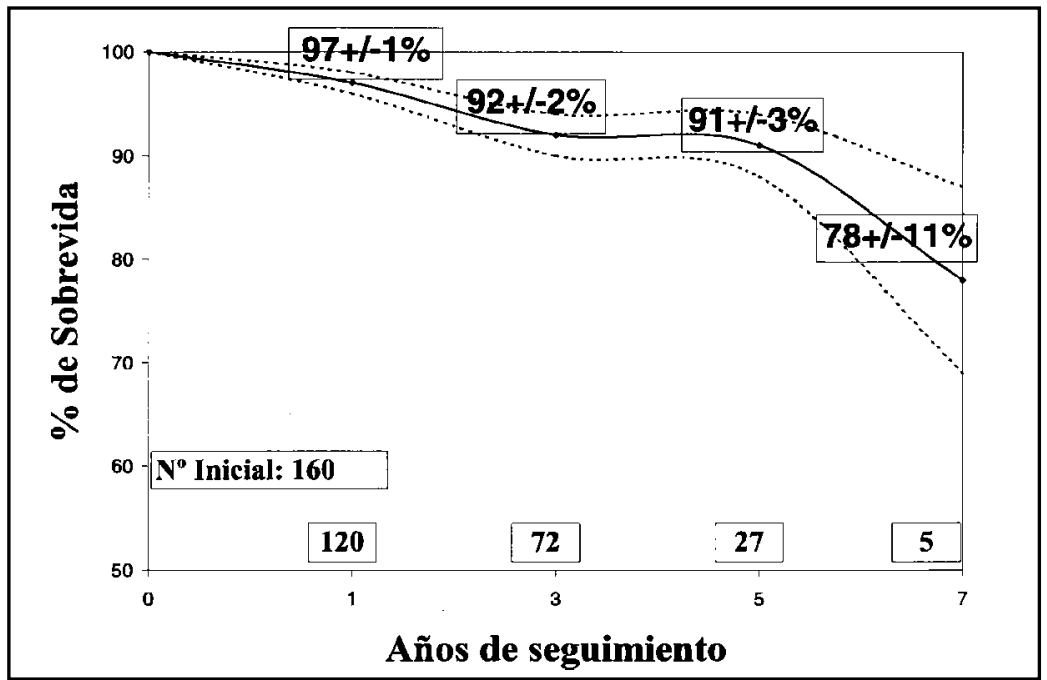

FiguRA 3. Sobrevida libre de hemorragia 


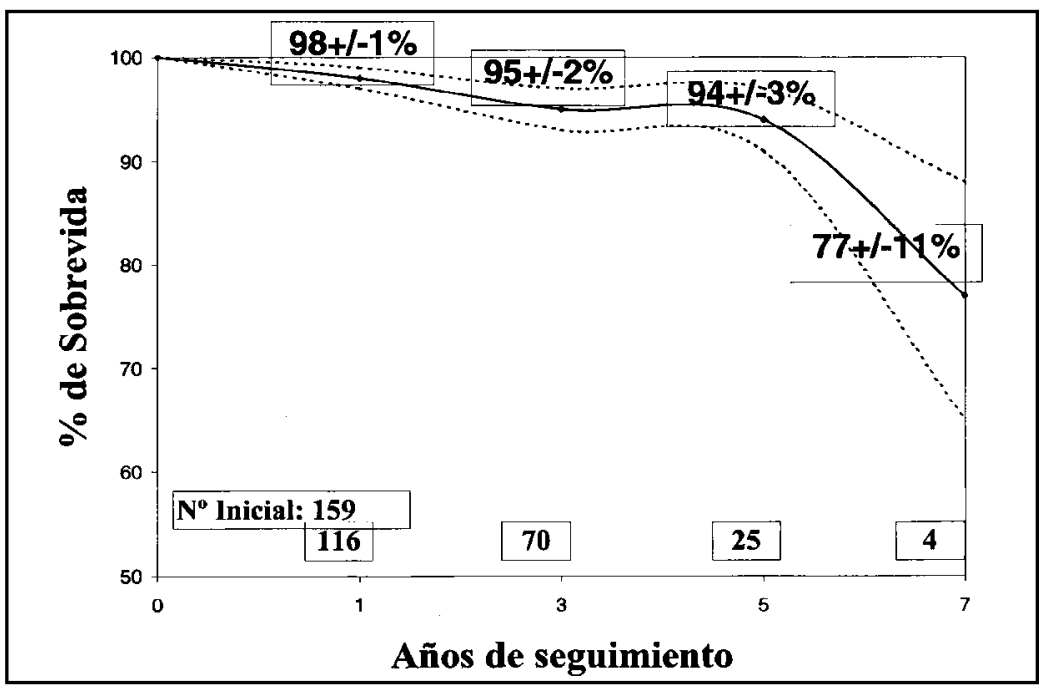

FigURA 4. Sobrevida libre de tromboembolismo

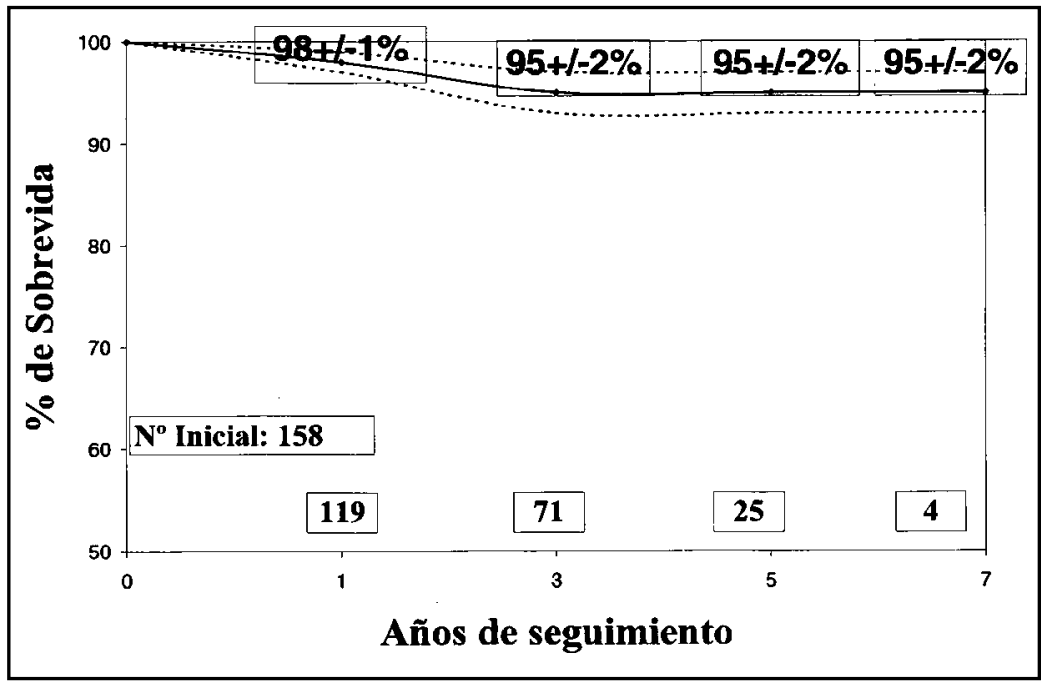

FIgURA 5. Sobrevida libre de endocarditis

\section{DisCUSIÓN}

Destaca entre las caracteństicas demográficas de nuestra serie la alta proporción de pacientes en capacidad funcional III o IV (73\%). Está demostrado que el RVA debe ser efectuado con estenosis, insuficiencia 0 doble lesión aórtica severa apenas aparezcan los síntomas, para así evitar la hipertrofia, dilatación y finalmente falla del ventrículo izquierdo. Si el paciente tiene síntomas es claro que el RVA los mejora, pero más importante aún, aumenta la sobrevida 6 . Por lo tanto, es evidente que queda mucho trabajo por hacer para aumentar la cantidad de pacientes operados en capacidad funcional II y así disminuir la morbimortalidad de este tipo de patología.

Las etiologías más frecuentes en nuestro grupo fueron la congénita (bicúspide), degenerativa, reumática y endocarditis. Esto contrasta con lo encontrado por Iung, en Europa, en un estudio de 92 centros en 25 países $^{7}$. La etiología degenerativa (74\%) fue claramente la más frecuente, sin embargo, los porcentajes de causa 
Tabla 7. Factores predictores de eventos en el seguimiento (tromboembolismo, hemorragia y endocarditis)

\begin{tabular}{|c|c|c|c|c|}
\hline \multicolumn{5}{|c|}{ Análisis univariado: Kaplan-Meier, Log Rank } \\
\hline Endocarditis infecciosa & $\begin{array}{l}\text { No } \\
\text { Sí }\end{array}$ & $\begin{array}{r}12 \\
4\end{array}$ & $\begin{array}{r}147 \\
11\end{array}$ & 0,010 \\
\hline Cirugía de urgencia & $\begin{array}{l}\text { No } \\
\text { Sí }\end{array}$ & $\begin{array}{r}11 \\
5\end{array}$ & $\begin{array}{r}126 \\
15\end{array}$ & 0,022 \\
\hline \multicolumn{5}{|c|}{ Análisis de múltiples variables: regresión Cox } \\
\hline Variable & Valor de $\mathrm{p}$ & $\operatorname{EXP}(ß)$ & Inferior & Superior \\
\hline Endocarditis infecciosa & 0,001 & 10 & 2,64 & 12,24 \\
\hline
\end{tabular}

reumática (12\%), congénita (7\%) y endocarditis (3\%) aparecen notoriamente más bajos que en nuestra muestra. Posibles explicaciones a estas diferencias son el mayor envejecimiento de la población, la disminución más temprana que tuvo la enfermedad reumática $\mathrm{y}$, tal vez, a una detección más precoz de los casos congénitos en el caso del estudio europeo.

Es notable que la mortalidad operatoria global para el reemplazo valvular aórtico con prótesis $4,6 \%$ es comparable a la publicada en grandes series norteamericanas. Astor encontró que 23.319 pacientes adultos (estudio de 900 hospitales no federales en Estados Unidos de Norteamérica [EE.UU]), tuvieron una mortalidad de $4,5 \%^{8}$. Jamieson describió resultados similares con una mortalidad global de $4,5 \%$ en 26.317 casos de RVA extraídos de la base de datos de la STS 9 . Estos datos se refieren a RVA sin revascularización miocárdica asociada, dado que es reconocido que el riesgo se incrementa en presencia de enfermedad coronaria significativa ${ }^{8}$. En cuanto a resultados de países en vías de desarrollo, las publicaciones son escasas. Antunez, en Sudáfrica, reportó una mortalidad 9,3\%. Sin embargo, esto corresponde a una serie no homogénea, con $28 \%$ de cirugías asociadas, por lo que es esperable mejores resultados en el caso del RVA como procedimiento aislado ${ }^{4}$.

Distinto es el caso en nuestra serie en la cirugía de urgencia, donde observamos una mortalidad elevada (16\%). Todos los factores de riesgo que están presentes en el análisis univariado de la mortalidad operatoria, corresponden al paciente tipo que ingresa a nuestro servicio para operarse de urgencia, con endocarditis activa, insuficiencia aórtica, inestabilidad hemodinámica y falla renal. Lamentablemente, la mayor parte de los pacientes referidos a nuestro servicio para tratamiento quirúrgico, han sido tratados médicamente (antibióticos, diuréticos, etc.), aun en presencia de lesiones valvulares hemodinámicamente significativas y que producen insuficiencia cardíaca. Estos pacientes deben ser operados de todas maneras y no hay ventajas en intentar sesterilizar» las válvulas antes del reemplazo valvular. El riesgo operatorio de un paciente con endocarditis sin falla renal o compromiso de otros órganos, es similar al de una operación electiva. La presencia de disfunción renal en un paciente con endocarditis (asumiendo que se ha monitorizado la administración de antibióticos con niveles plasmáticos), generalmente indica persistencia de la infección de manera que debería considerarse como una razón para referir un paciente para tratamiento quirúrgico antes que su función renal se deteriore más o que se asocie a otras complicaciones ${ }^{10-12}$. Por otro lado, si bien nuestro análisis de múltiples variables muestra que la endocarditis es un factor significativo e influyente en la mortalidad, la insuficiencia renal lo es más, lo que nos obliga a focalizar nuestros esfuerzos terapéuticos en los casos en que existe ya esta complicación. A este respecto, la hemodiafiltración 
continua temprana ha demostrado que es útil y debiera ser considerada, sobre todo en pacientes con inestabilidad hemodinámica ${ }^{13}$.

Las complicaciones del tratamiento anticoagulante son mayores que las publicadas en la literatura internacional. Akins revisó diversos estudios que totalizan 6.027 años-paciente con la prótesis de Medtronic Hall en posición aórtica, encontrando que la tasa lineal de eventos hemorrágicos fue de 0,9\%, con una variación según el estudio entre $0,7 \%$ y 1,7\%. Esto está bastante por debajo del 3,29\% que encontramos en nuestra serie. En relación al tromboembolismo, los resultados no son tan ominosos, ya que nuestra tasa fue de 2,04\%. Sin embargo, aún está por sobre el promedio de 1,4\% encontrado por Akins en estudios que incluyen 9.443 años-paciente. $\mathrm{Si}$ tomamos en cuenta el índice compuesto de hemorragia más tromboembolismo, que básicamente nos dicta el riesgo total del tratamiento anticoagulante, nos encontramos que tenemos $5,33 \%$, que dista mucho del $2,77 \%$ de las series que revisó el autor ya mencionado para las prótesis de Medtronic Hall ${ }^{14}$. Un factor que puede influir en estos resultados es el hecho de que nuestros pacientes fueron tratados con acenocumarol y no con warfarina como en las series norteamericanas. Esto puede influir en la estabilidad del INR y, por ende, en el desarrollo de trombosis y hemorragia. Desafortunadamente, nuestros pacientes tienen todos los problemas inherentes al subdesarrollo para acceder a un control adecuado: bajo nivel de escolaridad, falta de dinero, dificultad para acceder a atención en los centros de atención públicos, que se traducen en horas o días perdidos. Esto tiene implicancias laborales y familiares. A eso se agrega que puede pasar mucho tiempo con un control inadecuado 0 inexistente sin que ocurran problemas, lo que es probable que se traduzca en una menor adherencia a los controles y al tratamiento.

Cuando ocurren complicaciones, pueden presentarse en forma súbita y lamentablemente pueden ser catastróficas y dejar secuelas graves. La única publicación que hemos encontrado sobre resultados alejados con prótesis mecánica en el medio nacional, cubre un período bastante más largo y una población seleccionada de mayores recursos económicos que esta ${ }^{15}$. Al restringirnos a un período más limitado y reciente, la serie es más homogénea y representativa de lo que ocurre actualmente. Esto es reforzado al no incluir pacientes en quienes se les efectuó otro procedimiento cardíaco en la misma operación.

Creemos que estos resultados hacen necesario identificar los aspectos que se pueden optimizar considerando las peculiaridades locales. Con respecto a las complicaciones hemorrágicas, hay por lo menos tres factores modificables: el primero es tratar de mantener un INR entre 2,0 y 2,5, más bajo del que históricamente se ha considerado necesario en pacientes con prótesis mecánicas ${ }^{16,17}$. En segundo lugar, quizás más importante que el anterior, evitar las variaciones extremas en el nivel de anticoagulación, lo que se consigue con un control periódico más frecuente y acucioso ${ }^{18}$. Esto se puede implementar en policlínicos generales con sistemas modernos más simples ${ }^{18}$ y ajuste de dosis con sistemas automatizados. Por último, asociar dosis bajas de aspirina y mantener niveles más bajos de anticoagulación sin que esto signifique un aumento del tromboembolismo ${ }^{20}$. El uso de aspirina exclusiva ( $\sin$ tratamiento anticoagulante asociado) ha sido reportado en forma esporádica en el pasado y es motivo de estudios prospectivos. Seguramente el costo-beneficio de estas medidas será ampliamente favorable al evitar la morbilidad y secuelas del tromboembolismo y hemorragias.

También hay que considerar las alternativas al reemplazo valvular con prótesis mecánica: xenoinjertos, homoinjertos aórticos y autoinjertos pulmonares (operación de Ross). Especialmente en estos últimos, las ventajas en calidad de vida son muy importantes: ausencia de tromboembolismo y mejor hemodinamia que se traduce en normalización de la masa ventricular izquierda. Es posible que en esta población con múltiples problemas sociales esto se traduzca en una mejor sobrevida. Seguramente un número importante de pacientes al ser enfrentado a la disyuntiva del tratamiento anticoagulante y los problemas que hemos visto prefieran la posibilidad de una vida sin restricciones y el riesgo de una eventual reoperación en el largo plazo.

La afirmación de que las prótesis mecánicas son una solución definitiva no es correcta. Existe un porcentaje significativo y constante de pacientes que son reoperados. Muchas veces esto es por un problema grave como endocarditis o trombosis de la prótesis y el riesgo de la reoperación es considerablemente más alto que el de una reope- 
ración en alguna de las alternativas mencionadas arriba que generalmente se deterioran en forma lenta ${ }^{23}$. La incidencia de reoperación por endocarditis protésica en esta serie fue sólo discretamente mayor que la reportada en la literatura internacional24,25. Esto podría explicarse por el menor cuidado que tienen nuestros pacientes frente a enfermedades comunes, déficit nutricional, falta de higiene dental y quizás manejo no óptimo en las múltiples tomas de muestras de sangre para controlar el tratamiento anticoagulante. Además, existen otras causas de reoperación entre las que se cuentan las demostradas en las series de la

\section{REFERENCIAS}

1. Topol E. Anticoagulation With Prosthetic Cardiac Valves. Arch Intern Med 2003; 163: 2251-2.

2. Akins C. Results with mechanical cardiac valvular prostheses. Ann Thorac Surg 1995; 60: 1836-44.

3. BirKMeyer N, BirKMEYer J, Tosteson A, Grunkemeier G, MARRIN C, O'Connor G. Prosthetic valve type for patients undergoing aortic valve replacement: a decision analysis. Ann Thorac Surg 2000; 70: 1946-52.

4. Antunes M, Wessels A, Sadowski, Schutz J, VanderDONK K, Oliveira J ET aL. Medtronic-Hall valve replacement in a third world population group. A review of the performance of 1000 prostheses. J Thorac CV Surg 1988; 95: 880-91.

5. Edmunds L Jr, Ciark R, Cohn L, Grunkemeier G, Miuer C, Weisel R. Guidelines for Reporting Morbidity and Mortality After Cardiac Valvular Operations. Ann Thorac Surg 1996; 62: 932-5.

6. Bonow R, Carabello B, De León A, Edmunds L JR, FedderLy B, Freed M et al. ACC/AHA Task Force Report ACC/AHA Guidelines for the Management of Patients with Valvular Heart Disease. JACC 1998; 32: 1486-588.

7. Iung B, Baron G, Butchart E, Delahaye F, GohlKeBärwolf C, LeVANG O ET AL. A prospective survey of patients with valvular heart disease in Europe: The Euro Heart Survey on Valvular Heart Disease. Eur Heart J 2003; 24: 1231-43.

8. Astor B, Kaczmarek R, Hefruin B, Daley R. Mortality after Aortic Valve Replacement: results from a nationally representative database. Ann Thorac Surg 2000; 70: 1939-45.

9. Jamieson W, Edwards F, Schwartz M, Bero J, Clark $\mathrm{R}$, GROver F. Risk stratification for cardiac valve
Clínica Mayo ${ }^{26}$ : formación de pannus, mismatch, dehiscencia protésica sin infección y falla estructural en prótesis de bola, lo que nos aleja del concepto de que las prótesis mecánicas son una solución permanente.

En conclusión, el RVA tiene baja mortalidad global y en cirugía electiva. La sobrevida a mediano plazo es buena, pero existe una tasa mayor de complicaciones del tratamiento anticoagulante que la publicada en países desarrollados. La insuficiencia renal y la endocarditis son los principales factores asociados a mortalidad $\mathrm{y}$ complicaciones inmediatas y tardías.

replacement. National Cardiac Surgery Database. Database Committee of The Society of Thoracic Surgeons. Ann Thorac Surg 1999; 67: 943-51.

10. DuRACK D. Evaluating and optimizing outcomes of surgery for endocarditis. JAMA 2003; 290: 3250-1.

11. Middiemost S, Wisenbaugh T, Meyerowitz C, TeEger S, Essop R, Skoutarigis J ET AL. A case for early surgery in native left sided endocarditis complicated by heart failure. J Am Coll Cardiol 1991; 18: 663-78.

12. Moon M, Stinson E, Miuer C. Surgical Treatment of Endocarditis. Prog CV Diseases 1997; 40: 239-64.

13. Bent P, Tan H, Belomo R, Buckmaster J, Doolan L, HART G ET AL. Early and intensive continuous hemofiltration for severe renal failure after cardiac surgery. Ann Thorac Surg 2001; 71: 832-7.

14. AkINs W. Long-Term Results with the Medtronic Hall Valvular Prosthesis. Ann Thorac Surg 1996; 61: 806-13.

15. Braun S, Irarrázaval M, Corbalán R, Chamorro G, Dubernet J, Maturana G et al. Evolución alejada del reemplazo valvular aórtico con prótesis mecánica: experiencia con prótesis de Starr-Edwards (1965-1974) y Bjork Shiley (1973-1981). Rev Méd Chile 1990; 118: 1355-61.

16. Skudicky D, Esopp M, Wisenbagh T, Skoularigis J, Essop AR, DuLAbh A et AL. Frequency of prosthetic valve related complications with very low level warfarin anticoagulation associated with dypiridamol alter valve replacement with Saint Jude Medical prostheses. Am J Cardiol 1994; 74: 113741.

17. Cannegieter S, Rosendaal F, Wintzen A, Van Der Meer F, Vandenbroucke J, Briet E et al. Optimal oral anticoagulant therapy in patients with mechanical heart valves. N Engl J Med 1995; 333: 11-7. 
18. Butchart E, Payne N, Li H, Buchan K, Mandana K, GRUNKEMEIER G. Better anticoagulation control improves survival after valve replacement. J Thorac Cardiovasc Surg 2002; 123: 715-23.

19. Koertke $H$, Minami $K$, Boethig D, Breymann $T$, Seifert D, Wagner O ET aL. INR Self-Management Permits Lower Anticoagulation Levels After Mechanical Heart Valve Replacement. Circulation 2003; 108 (suppl II): II-75-II-78.

20. Turpie A, Gent M, Laupacis A, Latour Y, Gunstensen J, BASILE F ET AL. A comparison of aspirin with placebo in patients treated with warfarin after heart-valve replacement. N Engl J Med 1993; 329: 524-9.

21. Jones M, O’Kane H, Gladstone DJ, Sarsam MA, Campalani G, Macgowan S et al. Repeat heart valve surgery: risk factors for operative mortality. J Thorac Cardiovasc Surg 2001; 122: 913-18.

22. Vlessis A, Khaki A, Grunkemeier GL, L H, Starr A. Risk, diagnosis and management of prosthetic valve endocarditis: a review. J Heart Valve Dis 1997; 6: 443-65.

23. Васн D. Choice of Prosthetic Heart Valves: Update for the Next Generation. J Am Coll Cardiol 2003; 42: 1717-9.

24. Grover F, David C, Charles O, Wiluam H, Gulshan S, HAMMERMEISTER K. Determinants of the occurrence of and survival from prosthetic valve endocarditis. Experience of the Veterans Affairs Cooperative Study on Valvular Heart Disease. J Thorac Cardiovasc Surg 1994; 108: 207-14.

25. Edwards M, Ratnatunga C, Dore C, Taylor K. Thirty-day mortality and long-term survival following surgery for prosthetic endocarditis: a study from the UK heart valve registry. Eur J Cardiothorac Surg 1998; 14: 156-64.

26. Potter D, Sundt T, Zehr K, Dearani JA, Daly R, Mulany C ET AL. Operative risk of reoperative aortic valve replacement. J Thorac Cardiovasc Surg 2005; 129: 94-103.

\section{Apéndice. Variables codificadas en el análisis uni y multivariado}

\author{
Variables \\ Edad \\ Fecha de operación \\ Sexo \\ Vive dentro o fuera de la región metropolitana \\ IMC (índice de masa corporal) \\ Obeso (IMC >30) \\ Desnutrido (IMC $<20)$ \\ Tabaquismo \\ Diabetes \\ Insuficiencia renal crónica \\ Creatinina \\ Hipercolesterolemia \\ Hipertensión arterial
}

Accidente vascular encefálico

Endocarditis infecciosa

Enf bronquial obstructiva crónica

Cirugía cardíaca previa

Fibrilación auricular

Capacidad funcional (NYHA)

Historia de insuficiencia cardíaca

Historia de infarto previo

Uso inótropos

Fracción de eyección

Dimensión ventricular sistólica-diastólica

Estenosis, insuficiencia o enfermedad aórtica

Carácter de la operación: electiva o urgente 